\title{
SERIES SOLUTIONS FOR THE PROFILES OF AXISYMMETRIC FLUID-LIQUID INTERFACES
}

\author{
WiLfRED JAMES PinCHIN
}

When a solid meets the interface between two fluids, the resulting surface may assume a variety of shapes, depending on certain characteristics of the solid, the contiguous fluids, and the net body force, gravity. If the solid is rotationally symmetric and has its axis vertical, then the resulting meniscal surface is said to be axisymmetric.

Truncated series approximations, describing the profiles and other properties of axisymmetric menisci, have been in use since the beginning of the nineteenth century, when the governing differential equations were first formulated by Young and Laplace. In recent years it has become popular to produce tabulated solutions of meniscus profiles using numerical algorithms with computer assistance.

This thesis commences its analytic presentation with the derivation of recurrence relations for the coefficients of forward and reverse Taylor series in Cartesian coordinates, valid in the neighbourhood of the vertex of a sessile or pendant drop. Elementary series analysis techniques are used to obtain information describing the dominant singularities of the forward series, facilitating their removal. The modified series have improved convergence properties. The techniques of stretching and straining of variables are used in deriving forward and reverse perturbation series, also in Cartesian coordinates, for small sessile and pendant drops. Asymptotic approximations for the characteristic dimensions of the drops are easily obtained from the perturbation series for these

Received 27 October 1982. Thesis submitted to University of Newcastle, March 1982. Degree approved July 1982. Supervisors: Dr W.T.F. Lau, Dr W. Wood and Dr S. McElwain. 
profiles.

A pair of matching perturbation series is obtained in reverse form to describe large external menisci. One of these series is obtained by an iteration method.

The singular perturbation series are parameter expansions, except the series derived by iteration, which must be considered a coordinate expansion. Under certain conditions of distribution, the families of profiles exhibit envelope curves. The equations for three of these envelopes are obtained by exploiting the straining equations.

It is argued that both the systematic development of techniques, and the interrelationships of the ensuing series, are of interest in their own right. Numerically, the series generate accurate profile points, offering an alternative to the use of tabulated profiles within the domains of validity of the series. Furthermore, certain analytical characteristics of the solutions may be ascertained from the serics.

Avondale College,

Cooranboing, New South Wales 2265, Australia. 